DOI 10. 18307/2020. 0315

(c) 2020 by Journal of Lake Sciences

\title{
不同生态处理方法对黑臭河道底泥细菌多样性与群落结构的影响”
}

\author{
金宗安 ${ }^{1}$,刘泽君 ${ }^{1}$, 唐雅丽 ${ }^{1 * *}$, 关保华 ${ }^{2 * *}$, 刘正文 ${ }^{1,2}$, 任丽娟 1 \\ (1:暨南大学生态系与水生生物研究中心,广州 510632) \\ (2:中国科学院南京地理与湖泊研究所湖泊科学与环境国家重点实验室,南京 210008)
}

\begin{abstract}
摘 要: 底泥细菌代谢是城市河道底泥代谢物的主要来源, 最终决定城市河道的生态状况. 本文研究了黑臭河道底泥经 添加硝酸钙、生物促生剂和种植沉水植物处理后底泥中细菌群落结构的响应, 以期为城市黑臭河道细菌群落的改善和综 合治理提供理论依据. 实验结果表明: 经过不同生态处理后, 上覆水中, 添加硝酸钻组总氮 ( TN) 含量显著高于对照组, 添加生物促生剂组溶解氧浓度显著高于对照组. 沉积物中, 所有处理组的氧化还原电位值 (ORP) 均显著高于对照组, 种 植沉水植物组和添加硝酸䥻组 TN 含量均显著低于对照组, 沉积物理化性质得到一定改善. 对不同生态处理组底泥细菌 群落的研究发现, 处理组底泥细菌群落产生了较大变化, 且不同处理组细菌群落变化不同, 生物促生剂组底泥中细菌的 Sobs 指数和 Chao 1 指数显著高于对照组和硝酸钻组, 且生物促生剂组 Shannon 指数和 PD 指数显著高于硝酸钙组. Proteobacteria (Deltaproteobacteria、Betaproteobacteria、Gammaproteobacteria)、Chloroflexi、Firmicutes、Bacteroidetes 和 Spirochaetae 是 各实验组的主要优势菌门; 非度量多维尺度分析表明: 硝酸䥻和生物促生剂的投加可明显改变底泥细菌群落结构组成. 在属水平上, uncultured_Anaerolineaceae、Ferribacterium、uncultured_Xanthomonadales_Incertae_Sedis 是导致底泥细菌群落发 生变化的主要菌属. 穴余分析结果表明,底泥 ORP 的变化是驱动细菌群落结构变化的关键环境因素.
\end{abstract}

关键词: 黑臭河道; 硝酸钙; 生物促生剂;沉水植物; 细菌群落

\section{Effect of different ecological treatment methods on microbial community in black and odorous sediment of urban river*}

\author{
JIN Zongan ${ }^{1}$, LIU Zejun ${ }^{1}$, TANG Yali $^{1 * *}$, GUAN Baohua ${ }^{2 * *}$, LIU Zhengwen ${ }^{1,2}$ \& REN Lijuan ${ }^{1}$ \\ (1: Department of Ecology and Institute of Hydrobiology, Jinan University, Guangzhou 510632, P.R. China) \\ (2: State Key Laboratory of Lake Science and Environment, Nanjing Institute of Geography and Limnology, Chinese Academy \\ of Sciences, Nanjing 210008, P.R. China)
}

\begin{abstract}
Sediment microorganisms are the main source of sediment metabolism in urban rivers, which ultimately determine the ecological status of the rivers. In this paper, to analyze the effect of different ecological treatments on sediment microbial community, we established artificial mesocosms to mimic sediment status of the black-stinking river with different ecological treatments as calcium nitrate, bio-energizer addition, and submerged macrophytes transplantation. Illumina Miseq sequencing was applied to reveal the bacteria community variations of all treated and untreated sediments. The results showed that in the overlying water, the total nitrogen ( TN) content of calcium nitrate addition group was significantly higher than that in the control group, dissolved oxygen concentration in the bio-energizer addition group was significantly higher than that in the control group. In the sediment, the oxidation reduction potential (ORP) values of all the treatment groups were significantly higher than those in the control group; TN content of calcium nitrate addition group and submerged macrophytes transplantation group were both significantly lower than those in the control group, which indicate the improvement of sediment status of treatment group. Bacterial communities were also varied in treatment group, and showed diverse variations with different treatments. The Sobs and Chao 1 microbial diversity indices in the bio-energizer addition group were both significantly higher than those in the control group and calcium nitrate addition group; the Shannon and PD diversity indices for microbes in the bio-energizer addition sediment were both significantly higher than those in
\end{abstract}

* 2019-09-12 收稿;2019-10-24 收修改稿.

国家自然科学基金项目 (31000219) 资助.

** 通信作者; E-mail: litangyali@163.com, bhguan@ niglas.ac.cn. 
calcium nitrate addition group. All sediment microbial communities were dominated by Proteobacteria (Deltaproteobacteria, Betaproteobacteria, Gammaproteobacteria), Chloroflexi, Firmicutes, Bacteroidetes and Spirochaetae phyla. Nonmetric multidimensional scaling analysis showed that the addition of calcium nitrate and bio-energizer can significantly alter the composition of bacterial community structure. Uncultured_Anaerolineaceae, Ferribacterium and uncultured_Xanthomonadales_Incertaeae_Sedis were the main genus causing variations in the bacterial community. Redundancy analysis indicated that the change of sediment ORP is a key environmental factor driving the variation of bacterial community structure.

Keywords: Black-stinking river; calcium nitrate; bio-energizer; submerged macrophytes; microbial community

沉积物是河流的重要组成部分, 是各种人为污染物如重金属、营养物和有机物等 ${ }^{[1]}$ 的汇. 沉积物中污染 物的降解消耗大量溶解氧导致厌氧微生物大量繁殖, 厌氧微生物分解有机物, 产生大量的恶臭气体, 并形成 一些黑色物质, 最终导致生物多样性锐减和生态功能的退化 ${ }^{[2]}$.

淡水沉积物中的细菌群落是有机物和营养物质的生物地球化学循环、污染物的生物降解和转化以及生 态系统健康维持的关键参与者之一 ${ }^{[3-4]}$. 水生态系统中营养盐、环境和污染状况的任何变化都将直接影响细 菌群落,进而影响营养盐循环和其他生物群落, 因此, 细菌群落可作为环境状况的评价指标 ${ }^{[5]}$. 研究沉积物 中细菌多样性和群落结构与其生境的变化关系, 对于开展黑臭河道的生态治理具有重要意义.

添加硝酸钻是沉积物处理的有效手段, 硝酸盐作为电子受体可被大量与有机物氧化相关的微生物还 原, 进而促进有机污染物的生物降解 ${ }^{[6]}$. 与硝酸盐相比, 添加生物促生剂可为微生物提供多种营养物质, 有 效促进土著微生物的增殖和活性, 同时抑制有害微生物的代谢和污染物的厌氧分解, 进而刺激和加速自然 生化反应 ${ }^{[7]}$. 种植沉水植物作为控制内源负荷的有效手段, 可提升沉积物的氧化还原电位及其对磷的滞留 能力 ${ }^{[8]}$, 减少沉积物中磷、铵和硝酸盐向上覆水释放 ${ }^{[9]}$. 然而, 这些研究通常侧重于沉积物及水体理化性质 的变化, 关于沉积物中的污染物与细菌群落之间的相互作用及其在水生态系统功能和服务中的作用研究相 对较少 ${ }^{[10]}$,细菌群落对污染物的响应和恢复力方面的研究也较为缺乏 ${ }^{[11]}$.

本文应用人工模拟系统, 旨在探究黑臭河道沉积物经添加硝酸钙、生物促生剂以及种植沉水植物处理 后, 沉积物中细菌群落结构的变化, 明确不同细菌群落在黑臭河道处理中的作用, 确定导致底泥细菌群落结 构产生差异的主要物种, 为城市黑臭河道细菌群落的改善和综合治理提供理论依据.

\section{1 材料与方法}

\section{1 材料}

采集苏州市东山镇上的黑臭河道表层 $(0 \sim 20 \mathrm{~cm})$ 富含有机质且呈黑臭状态的底泥,用于后续实验; 实 验用水来源于南京地理湖泊研究所东太湖实验站附近的池塘, 呈黑臭状态. 生物促生剂由河南华宇环保科 技有限公司提供,富含营养物(氮、碳、磷等)、酶、有机酸、细胞分裂素、维生素和微量元素等物质.

\section{2 实验方法}

本实验共设置 4 个实验组, 分别为空白对照组 ( CK 组) 和 3 个处理组, 即沉水植物组 ( MC 组)、硝酸钲 组 ( CN 组)、生物促生剂组 ( $\mathrm{BE}$ 组), 每组设置 3 个平行组. 实验装置为 $30 \mathrm{~cm} \times 100 \mathrm{~cm}$ (管内径 $\times$ 高度) 的柱状 PVC 管, 第 0 天时, 混合所有底泥初始样并将其定义为原泥 (CS 组). 将采集的泥样做均一化处理后, 铺设 30 $\mathrm{cm}$ 厚度的原泥于实验装置中, 注人 $60 \mathrm{~cm}$ 上覆水. 静置 $2 \mathrm{~d}$ 后, 种植 1 株苦草 (Vallisneria natans) (株高: $24.0 \pm 0.5 \mathrm{~cm}$, 鲜重: $2.12 \pm 0.10 \mathrm{~g}$ /株) 于 $\mathrm{MC}$ 组,一周后开始实验. 在 $\mathrm{CN}$ 组沉积物表层往上 $15 \mathrm{~cm}$ 处一次性 注射 $45.3 \mathrm{~g} \mathrm{~N} / \mathrm{m}^{2}$ 的硝酸钻; 在相同位置将 $2.5 \mathrm{~mL} /\left(\mathrm{m}^{3} \cdot \mathrm{d}\right)$ 的生物促生剂分散注射于 BE 组; CK 组不做任 何处理. 实验装置置于池塘中不密封, 定期补充黑臭水以保持总体积. 实验于 2016 年 8-9 月间进行, 鉴于 生物促生剂、硝酸钙投加以及沉水植物种植对河流和沉积物的处理的现有相关研究持续时间为 10 $32 \mathrm{~d}^{[7,12-15]}$, 本实验选取实验周期为 $28 \mathrm{~d}$, 并于第 0 天和 28 天分别采集水样和表层 $5 \mathrm{~cm}$ 厚的沉积物样品用 于理化性质和细菌指标测试.

实验开始时 (第 0 天), 各实验组主要水质指标为: $\mathrm{pH}: 8.60 \pm 0.11$, 氧化还原电位 (ORP) : $184 \pm 11.75$ $\mathrm{mV}$, 溶解氧 ( DO ) : $6.49 \pm 0.93 \mathrm{mg} / \mathrm{L}$, 总氮 $(\mathrm{TN}): 2.25 \pm 0.33 \mathrm{mg} / \mathrm{L}$, 总磷 $(\mathrm{TP}): 0.21 \pm 0.07 \mathrm{mg} / \mathrm{L}$; 底泥中各指 标为: $\mathrm{pH}: 6.92 \pm 0.03, \mathrm{ORP}: 18.24 \pm 4.08 \mathrm{mV}, \mathrm{TN}: 3.97 \pm 0.60 \mathrm{mg} / \mathrm{g}, \mathrm{TP}: 3.00 \pm 0.83 \mathrm{mg} / \mathrm{g}$, 总有机碳 (TOC) : 
$36.46 \pm 6.43 \mathrm{mg} / \mathrm{g}$.

\section{3 样品相关测定方法}

1.3.1 沉积物理化指标测定 沉积物样品 $\mathrm{pH} 、 \mathrm{ORP}$ 采用 FJA-6 型氧化还原电位去极化全自动测定仪测定; TN、TP 含量采用元素分析仪 (EA3000, Italy, Euro Vector) 测定, TOC 含量通过电感耦合等离子体发射光谱仪 (American, Leeman, prodigy) 测定.

1.3.2 细菌多样性指数测定 沉积物样品经预处理后, 由上海美吉生物医药科技有限公司 (简称美吉) 的 Illumina Miseq 平台测序, 利用引物 338F/806R 扩增细菌 16S rRNA 基因 V3 V4 区, 用 2\%琼脂糖凝胶电泳 检测 PCR 产物.

\section{4 生物信息学及统计分析}

数据分析使用美吉公司提供的 I-Sanger 平台进行. 采用 Mothur 软件对 $97 \%$ 相似水平的 OTU 进行 Alpha 多样性分析. 采用 R 语言的 vegan 包进行样本的热图分析 (Heapmap)、非度量多维尺度 (NMDS) 分析和圥余 分析 (RDA). 采用 R 语言的 stats 包和 Python 的 scipy 包进行 One-way ANOVA 分析.

\section{2 结果}

\section{1 水体及沉积物理化性质分析}

经过 $28 \mathrm{~d}$ 处理后, $\mathrm{CN}$ 组上覆水中 $\mathrm{TN}$ 含量显著高于 $\mathrm{CK}$ 组 $(P<0.05$, 表 1), 各处理组 DO ORP 均高于 $\mathrm{CK}$ 组, 其中, $\mathrm{BE}$ 组 DO 含量显著高于 $\mathrm{CK}$ 组 $(P<0.05)$. 沉积物中 3 组处理组 ORP 均显著高于 $\mathrm{CK}$ 组 $(P<$ $0.05)$; $\mathrm{MC}$ 组和 $\mathrm{CN}$ 组 $\mathrm{TN}$ 含量显著低于 $\mathrm{CK}$ 组和 $\mathrm{BE}$ 组 $(P<0.05)$; MC 组 TP 含量比 $\mathrm{CK}$ 组降低了 $5.80 \%$, 而 $\mathrm{CN}$ 组和 $\mathrm{BE}$ 组 $\mathrm{TP}$ 含量较 $\mathrm{CK}$ 组分别升高了 $1.90 \%$ 和 $4.61 \%$; TOC 含量从大到小依次为 $\mathrm{BE}$ 组 $>\mathrm{CK}$ 组 $>\mathrm{CN}$ 组 $>\mathrm{MC}$ 组. 此外, 与实验开始时相比, 实验结束时苦草株高 (35.83 $\pm 2.36 \mathrm{~cm})$ 、鲜重 ( $4.10 \pm 1.13 \mathrm{~g} /$ 株) 和株数 $(2.67 \pm 0.58$ 株) 均显著增加 $(P<0.05)$.

表 1 实验结束时不同实验组上覆水和沉积物样品的理化性质*

Tab.1 Physicochemical properties of water and sediment samples after 28 days of treatment

\begin{tabular}{cccccc}
\hline 上覆水 & $\mathrm{pH}$ & $\mathrm{ORP} / \mathrm{mV}$ & $\mathrm{TN} /(\mathrm{mg} / \mathrm{L})$ & $\mathrm{TP} /(\mathrm{mg} / \mathrm{L})$ & $\mathrm{DO} /(\mathrm{mg} / \mathrm{L})$ \\
\hline $\mathrm{CK}$ 组 & $8.68 \pm 0.16^{\mathrm{a}}$ & $170 \pm 22.61^{\mathrm{a}}$ & $1.99 \pm 0.40^{\mathrm{a}}$ & $0.37 \pm 0.07^{\mathrm{a}}$ & $5.98 \pm 0.27^{\mathrm{a}}$ \\
$\mathrm{MC}$ 组 & $8.69 \pm 0.25^{\mathrm{a}}$ & $183 \pm 1.00^{\mathrm{a}}$ & $1.76 \pm 0.21^{\mathrm{a}}$ & $0.26 \pm 0.16^{\mathrm{a}}$ & $6.81 \pm 1.39^{\mathrm{ab}}$ \\
$\mathrm{CN}$ 组 & $8.57 \pm 0.13^{\mathrm{a}}$ & $183 \pm 8.66^{\mathrm{a}}$ & $3.67 \pm 0.61^{\mathrm{b}}$ & $0.18 \pm 0.03^{\mathrm{a}}$ & $7.17 \pm 0.60^{\mathrm{ab}}$ \\
$\mathrm{BE}$ 组 & $8.60 \pm 0.25^{\mathrm{a}}$ & $190 \pm 3.46^{\mathrm{a}}$ & $1.90 \pm 0.18^{\mathrm{a}}$ & $0.28 \pm 0.17^{\mathrm{a}}$ & $7.67 \pm 0.14^{\mathrm{b}}$ \\
\hline 沉积物 & $\mathrm{pH}$ & $\mathrm{ORP} / \mathrm{mV}$ & $\mathrm{TN} /(\mathrm{mg} / \mathrm{g})$ & $\mathrm{TP} /(\mathrm{mg} / \mathrm{g})$ & $\mathrm{TOC} /(\mathrm{mg} / \mathrm{g})$ \\
\hline $\mathrm{CK}$ 组 & $7.75 \pm 0.05^{\mathrm{a}}$ & $4.17 \pm 2.11^{\mathrm{a}}$ & $3.69 \pm 0.35^{\mathrm{a}}$ & $2.07 \pm 0.07^{\mathrm{a}}$ & $32.36 \pm 1.07^{\mathrm{a}}$ \\
$\mathrm{MC}$ 组 & $7.67 \pm 0.18^{\mathrm{a}}$ & $23.90 \pm 1.84^{\mathrm{b}}$ & $3.22 \pm 0.12^{\mathrm{b}}$ & $1.95 \pm 0.21^{\mathrm{a}}$ & $29.69 \pm 2.03^{\mathrm{a}}$ \\
$\mathrm{CN}$ 组 & $7.74 \pm 0.07^{\mathrm{a}}$ & $65.00 \pm 1.39^{\mathrm{c}}$ & $3.25 \pm 0.26^{\mathrm{b}}$ & $2.11 \pm 0.19^{\mathrm{a}}$ & $29.75 \pm 2.40^{\mathrm{a}}$ \\
$\mathrm{BE}$ 组 & $7.61 \pm 0.12^{\mathrm{a}}$ & $68.03 \pm 4.09^{\mathrm{c}}$ & $3.46 \pm 0.06^{\mathrm{a}}$ & $2.17 \pm 0.08^{\mathrm{a}}$ & $32.71 \pm 0.84^{\mathrm{a}}$ \\
\hline
\end{tabular}

* 数据为平均值土标准偏差, 不同字母标记的数值表示在 $5 \%$ 的显著性水平下有显著性差异.

\section{2 不同生态处理方法对细菌多样性的影响}

利用 Illumina MiSeq 测序技术研究不同实验组底泥细菌的多样性. 共检测到 559332 条高质量序列, 平 均长度为 $440 \mathrm{bp}$, 覆盖度介于 $95.96 \%$ 96.34\% 之间; 不同实验组底泥共鉴定出 OTU 数量 6057 个, 结果涵盖 54 门, 128 纲, 259 目,445 科,781 属. 在 OTU 分析的基础上, 通过计算 Shannon-Wiener 指数、Simpson 指数、 PD (Phylogenetic diversity) 指数、Sobs 指数、Chao 1 和 ACE 丰富度对各实验组样本的多样性和丰富性进行评 价 (表 2). 结果显示: BE 组 Sobs 指数和 Chao 1 指数均显著高于 CK 组和 CN 组 $(P<0.05)$; 且 BE 组 Shannon 指数和 PD 指数显著高于 $\mathrm{CN}$ 组 $(P<0.05)$. 
表 2 不同实验组表层沉积物细菌多样性指数*

Tab.2 Microbial diversity index of surface sediments in different treatment groups

\begin{tabular}{ccccccc}
\hline 实验组 & Sobs 指数 & Shannon 指数 & Simpson 指数 & ACE 指数 & Chao 1 指数 & PD 指数 \\
\hline CS 组 & $3163 \pm 84.7^{\mathrm{a}}$ & $6.64 \pm 0.06^{\mathrm{ab}}$ & $0.0053 \pm 0.0008^{\mathrm{a}}$ & $4428.34 \pm 81.6^{\mathrm{ab}}$ & $4317.75 \pm 111.6^{\mathrm{ab}}$ & $234.2 \pm 6.97^{\mathrm{ab}}$ \\
$\mathrm{CK}$ 组 & $2977 \pm 191.2^{\mathrm{a}}$ & $6.54 \pm 0.11^{\mathrm{ab}}$ & $0.0058 \pm 0.0006^{\mathrm{a}}$ & $4172.06 \pm 264.4^{\mathrm{ab}}$ & $4075.22 \pm 215.6^{\mathrm{a}}$ & $218.4 \pm 12.93^{\mathrm{a}}$ \\
MC 组 & $3197 \pm 75.8^{\mathrm{a}}$ & $6.70 \pm 0.04^{\mathrm{a}}$ & $0.0049 \pm 0.0002^{\mathrm{a}}$ & $4367.70 \pm 69.4^{\mathrm{ab}}$ & $4255.30 \pm 34.8^{\mathrm{ab}}$ & $235.2 \pm 4.96^{\mathrm{ab}}$ \\
$\mathrm{CN}$ 组 & $2870 \pm 347.6^{\mathrm{a}}$ & $6.36 \pm 0.36^{\mathrm{b}}$ & $0.0086 \pm 0.0060^{\mathrm{a}}$ & $4043.02 \pm 479.7^{\mathrm{a}}$ & $3932.84 \pm 423.1^{\mathrm{a}}$ & $214.8 \pm 23.05^{\mathrm{a}}$ \\
BE 组 & $3301 \pm 103.2^{\mathrm{b}}$ & $6.76 \pm 0.11^{\mathrm{a}}$ & $0.0038 \pm 0.0005^{\mathrm{a}}$ & $4599.80 \pm 74.7^{\mathrm{b}}$ & $4514.42 \pm 37.5^{\mathrm{b}}$ & $244.7 \pm 5.34^{\mathrm{b}}$ \\
\hline
\end{tabular}

* 数据为平均值土标准偏差, 不同字母标记的数值表示在 $5 \%$ 的显著性水平下有显著性差异.

\section{3 不同生态处理方法对细菌群落组成的影响}

5 组底泥样品中细菌主要包括: Proteobacteria (39.53\% 41.68\%)、Chloroflexi ( 17.72\% 24.19\%)、 Firmicutes $(2.1 \% \sim 12.5 \%) 、$ Bacteroidetes $(4.65 \% \sim 7.91 \%)$ 和 Spirochaetae $(2.55 \% \sim 3.39 \%)$, 除上述 5 种优势菌 门外, OTU 平均相对丰度大于 $1 \%$ 的还有 Acidobacteria 、Latescibacteria、Aminicenantes、Nitrospirae、Chlorobi、Actinobacteria、Parcubacteria、TA06、 Candidate _ division _ OP3. 有 $1.54 \% \sim 2.55 \%$ 的序列不能在门一级进行分 类, 定义为 bacteria-unclassified, 将样本中丰度均值占 比均小于 $1 \%$ 的物种归为 others (图 1). Proteobacteria 和Chloroflexi 是各实验组中最丰富的门,在各组中无显 著差异 $(P>0.05)$; 在 Proteobacteria 中, Deltaproteobacteria (14.19\% 22.91\%) 、Betaproteobacteria ( 7.68\% 16.19\%) 和 Gammaproteobacteria $(7.72 \% \sim 13.25 \%$ ) 是所有实 验组中最丰富的纲. BE 组中 Firmicutes 显著低于其 他组 $(P<0.05)$; 相反, $\mathrm{BE}$ 组 Bacteroidetes 和 Spirochaetae 的相对丰度均显著高于其余各组 $(P<0.05)$.

Heatmap 图显示了底泥细菌群落前 30 属的物种

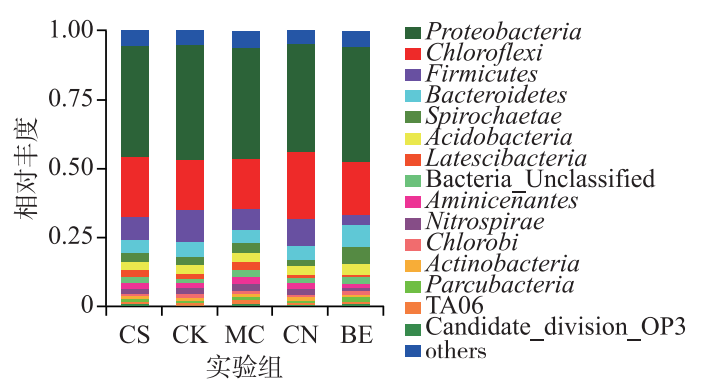

图 1 门分类水平上的细菌群落组成

Fig.1 Community composition of bacteria in the phylum level

组成 (图 2). 对各实验组最丰富的 5 属进行组间差异显著性检验分析 (one-way ANOVA), 结果表明: uncultured_Anaerolineaceae $(P<0.01) 、$ Ferribacterium $(P<0.01)$ 和 uncultured_Xanthomonadales_Incertae_Sedis $(P<$ $0.05)$ 是导致底泥细菌群落变化的主要菌属.

基于 Bray-Curtis 距离对不同实验组底泥细菌群落组成的 OTU 分布统计数据进行非度量多维尺度分析 (NMDS) 和 ANOSIM 分析, 结果显示: 不同生态处理方法显著改变了底泥群落组成 (ANOSIM, $r=0.4074$, $P=0.001)$; CK 组和 $\mathrm{MC}$ 组细菌群落结构组成较为类似; 而 $\mathrm{CN}$ 组和 $\mathrm{BE}$ 组与上述 2 组底泥细菌群落结构组 成具有明显差异 (图 3).

\section{4 细菌群落结构与环境因子的关系}

为进一步探究环境变量对底泥细菌群落结构的影响, 对相关参数进行 RDA 分析. 前两个轴分别解释了 底泥细菌群落结构变异的 $19.03 \%$ 和 10.69\%（图 4). 环境变量包括底泥的 $\mathrm{pH} 、 \mathrm{ORP} 、 \mathrm{TN} 、 \mathrm{TP} 、 \mathrm{TOC}$, 其中, ORP 是影响底泥细菌群落结构的关键环境因子 $(P<0.01)$.

\section{3 讨论}

\section{1 不同生态处理改变了上覆水和沉积物的理化特征}

沉积物原位修复技术通常用于控制沉积物中污染物的释放. 已有研究表明,在沉积物中添加硝酸钙可 有效提升沉积物的 ORP 值, 促进沉积物中 TOC 的降解 ${ }^{[6,13]}$. 同时, 硝酸盐钙的加人能固定铁磷和钙 磷 ${ }^{[13-14,16]}$, 降低水体中的 TP, 增加底泥 TP, 并减少底泥磷的释放. 另外, 在底泥中注人硝酸钙加速了反硝化 作用, 提高了细菌的活性, 使氮以气体形式排出, 进而提高了脱氮效果 ${ }^{[6,13-14,16]}$. 添加生物促生剂也可提升 


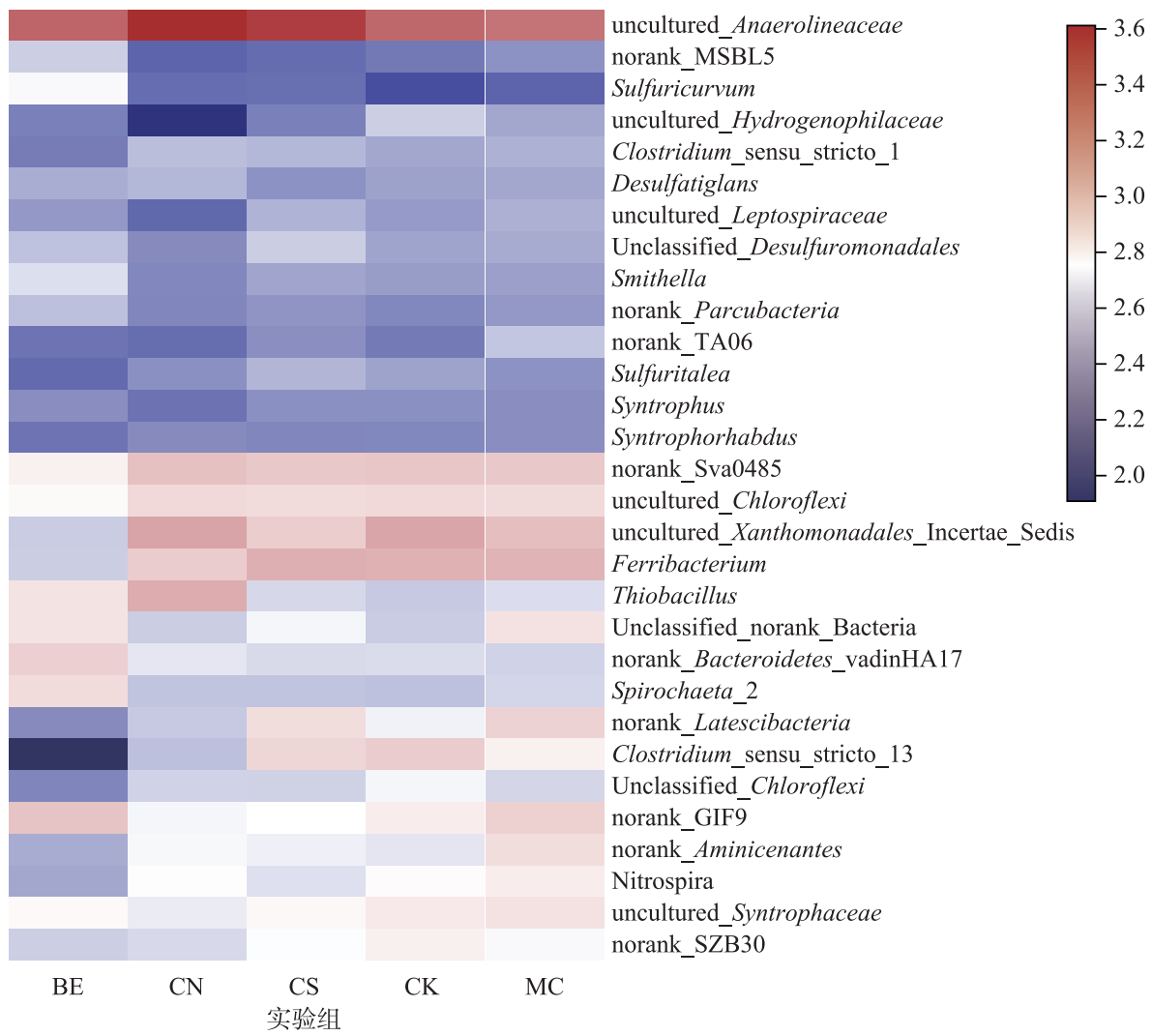

图 2 不同实验组中细菌群落前 30 属的热图分析(右侧颜色梯度(数值)表示各组属水平的平均相对丰度)

Fig. 2 Heatmap of the top 30 abundant genera in bacterial communities detected from different groups (the color of the spots in the right panel represents the mean relative abundance of the genera in each group)

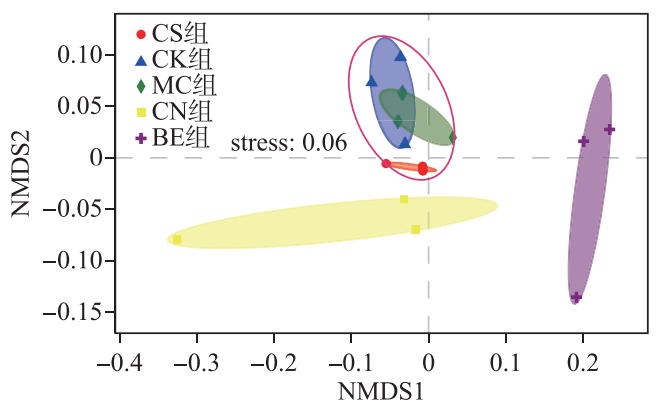

图 3 基于 Bray-Curtis 距离的 NMDS 分析

Fig.3 NMDS analysis based on

Bray-Curtis distance

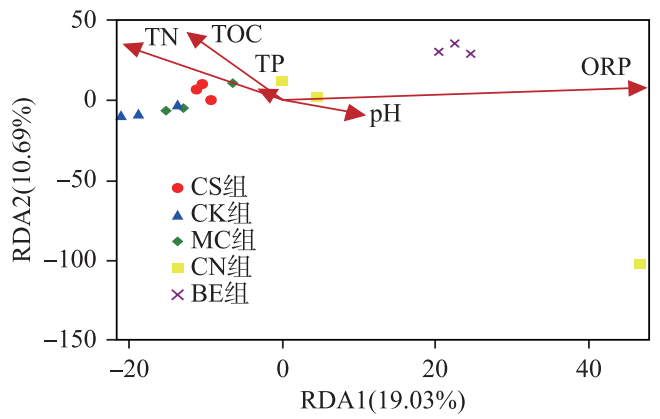

图 4 细菌群落组成和环境变量的几余分析

Fig.4 Redundancy analysis of bacteria community compositions and environmental variables

沉积物的 ORP 值和 DO 值, 通过细菌活性和多样性持续增加, 促进了其对碳的呼吸以及氮、磷的同化作用, 最终降低底泥 TOC 含量, 并使得水体中氮、磷元素固定在沉积物中 ${ }^{[7,17]}$. 种植沉水植物作为控制内源负荷的 有效手段, 可通过根际泌氧提升沉积物的 ORP 值 ${ }^{[8]}$, 并通过生长吸收氮、磷, 进而减少水体和沉积物中的 氮、磷 ${ }^{[8-9,18]}$. 
本实验中, 经过 $28 \mathrm{~d}$ 处理后, 仅 $\mathrm{BE}$ 组上覆水中 $\mathrm{DO}$ 浓度显著高于 $\mathrm{CK}$ 组, $\mathrm{CN}$ 组 $\mathrm{TN}$ 浓度显著高于 $\mathrm{CK}$ 组, 其他参数改善效果不明显, 这可能是由于处理时间较短所致. 但各处理组 ORP 和 DO 的平均值均高于 $\mathrm{CK}$ 组, 各处理组 TP 平均浓度均小于 CK 组; 除 CN 组外, MC 组和 BE 组 TN 平均浓度较 CK 组相比, 均有不 同程度下降,表现出一定的改善趋势.

沉积物中, 所有处理组的 ORP 值均显著高于对照组, 且 $\mathrm{CN}$ 组和 $\mathrm{BE}$ 组的 ORP 值显著高于 $\mathrm{MC}$ 组, MC 组和 $\mathrm{CN}$ 组的 TN 含量显著低于 $\mathrm{CK}$ 组. 这些参数表明底泥理化性质得到一定程度的改善. $\mathrm{CN}$ 组和 BE 组沉 积物 TP 含量虽然没有显著上升, 但其平均含量均高于对照组, 这两组水体 TP 平均含量的下降提示了这两 个处理组沉积物对水体磷的固定趋势. 与实验开始时相比, $\mathrm{MC}$ 组实验结束时苦草株高、鲜重和株数均显著 增加, MC 组水体和底泥 TN 和 TP 平均含量均有所下降. CN 组和 MC 组沉积物 TOC 平均含量也有下降, 这 与文献报道结果相似 ${ }^{[13-14,19]}$.

\section{2 不同生态处理方法改变了底泥细菌多样性}

基于 $16 \mathrm{~S} \mathrm{rRNA}$ 的 Miseq 测序表明, 处理组底泥细菌发生了很大的变化. 以大于 $97 \%$ 相似度 OTU 作为分 类单元计算, BE 组 Sobs 指数和 Chao 1 指数均显著高于 CK 组; BE 组 Shannon 指数和 PD 指数显著高于 CN 组. 这表明黑臭河道底泥细菌对不同生态处理方法的响应不尽相同,生物促生剂的投加可在一定程度上提 升底泥细菌多样性, 这可能是由于生物促生剂中富含多种营养素 (如酶、氨基酸、维生素等), 可有效促进细 菌的增殖并增加其活性 ${ }^{[7]}$. 研究发现沉积物注人硝酸钙后, 底泥细菌的多样性随温度升高呈下降趋势; 当 沉积物的温度为 25 或 $35^{\circ} \mathrm{C}$ 时, 底泥细菌的多样性甚至低于原底泥指标 ${ }^{[20]}$. 本实验期间, 水温介于 $23.24 \sim$ $30.26^{\circ} \mathrm{C}$ 之间, 在这一温度条件下, 某些物种的富集可能是导致 $\mathrm{CN}$ 组底泥细菌多样性降低的主要原因.

\section{3 不同生态处理方法显著改变了底泥细菌群落组成}

本研究中各实验组细菌群落结构与以往关于沉积物的研究结果基本一致 ${ }^{[4,21-22]}$. Proteobacteria 和 Chloroflexi 是各实验组中最丰富的门, 在各组中无显著差异; 本实验中, BE 组中 Firmicutes 显著低于其他组; Fir micutes 是粪便污染的重要指标, 它是识别人类粪便的标志之一 ${ }^{[23]}$. 研究表明, Firmicutes 是异养反硝化菌, 常 见于废水处理过程的反硝化生物反应器中 ${ }^{[24]}$, 其在 $\mathrm{CN}$ 组丰度较高可能是由于添加硝酸钙后反硝化作用增 强, 促使其转变为优势菌群. 生物促生剂的投加改变了底泥的还原性环境, 从而抑制了反硝化细菌及厌氧氨 氧化菌的活性, 这是其在 BE 组显著低于其他各组的主要原因 ${ }^{[12]}$. Wang 等 ${ }^{[25]}$ 的研究表明城市段河流中 Bacteroidetes 丰度较高的原因可能与居住区未经处理的排放废水中含有硝酸盐、氨、粪便等污染物有关; $\mathrm{BE}$ 组中 Bacteroidetes 丰度较高可能是由于生物促生剂富含多种营养素 (如碳、氮、磷等), 可向其提供丰富的营 养物质. 目前, 关于 Spirochaetae 菌门的生态生理功能的研究很少, Lee 等 ${ }^{[26]}$ 研究发现 Spirochaetae 可促进碳 水化合物和氨基酸发酵生成乙酸盐、氢气和二氧化碳; BE 组中 Spirochaetae 的相对丰度显著高于其余各组, 这表明底泥投加生物促生剂后可有效促进该菌门的生长和增殖.

细菌属水平的物种组间差异显著性检验分析表明: uncultured_Anaerolineaceae、Ferribacterium 和 uncultured_Xanthomonadales_Incertae_Sedis 是导致底泥细菌群落发生变化的主要菌属. Uncultured_Anaerolineaceae 是各样本中最丰富的属, 属于 Chloroflexi 门, 是典型的异养反硝化细菌, 在反硝化过程中可分解大分子 有机物产生甲烷和二氧化碳 ${ }^{[27]}$. Uncultured_Xanthomonadales_Incertae_Sedis 属于 Gammaproteobacteria 菌纲; 其常见于活性污泥中 ${ }^{[28]}$. 另一个丰富的属 Ferribacterium 属于 Betaproteobacteria 菌纲, 是一种铁还原菌; 先前 的研究表明, 在厌氧沉积物中, Ferribacterium 可将 $\mathrm{Fe}^{3+}$ 还原成 $\mathrm{Fe}^{2+[29]}, \mathrm{Fe}^{2+}$ 可与河道中有机物分解产生的 $\mathrm{H}_{2} \mathrm{~S}$ 反应生成 $\mathrm{FeS}$, 进而使底泥发黑. 因此, 我们推测 Ferribacterium 丰度降低(图 2), 其代谢产物 $\mathrm{Fe}^{2+}$ 的浓度 也会随之降低,进而有利于消除河道底泥黑臭现象.

基于 OTU 的 NMDS 和 ANOSIM 分析显示: 不同生态处理方法显著改变了底泥群落组成, MC 组和 CK 组的细菌群落组成较为相似, 而 $\mathrm{CN}$ 组和 $\mathrm{BE}$ 组与上述 2 组细菌群落组成具有明显差异. 这主要是由于底泥 经由硝酸钻和生物促生剂处理后, $\mathrm{CN}$ 组和 $\mathrm{BE}$ 组 $\mathrm{ORP}$ 值显著高于 $\mathrm{CK}$ 组和 $\mathrm{MC}$ 组所致.

\section{4 底泥氧化还原电位的变化是影响细菌群落结构的主要驱动因素}

$\mathrm{RDA}$ 分析结果表明, ORP 是影响底泥细菌群落组成的最重要因素. 有研究表明细菌的生长与 ORP 的变 化直接相关 ${ }^{[30]}$. 环境中的 ORP 很大程度上决定了该环境中细菌群落的代谢类型, 因此它们是生物活动的重 
要参数 ${ }^{[31-32]}$. 每种类型的微生物适应特定的 ORP 条件, 如厌氧土壤中微生物和酶活性与 ORP 呈负相关, 而 好氧微生物则需要更高的 ORP 值 ${ }^{[33-34]}$. Zeng 等 ${ }^{[35]}$ 对富营养化湖泊沉积物中细菌群落空间分布的研究结果 也表明, ORP 对沉积物中细菌群落组成具有显著影响,这也与本文的研究结果相一致.

\section{4 参考文献}

[ 1 ] Moreno B, Cañizares R, Macci C et al. Molecular tools to understand the bioremediation effect of plants and earthworms on contaminated marine sediments. Journal of Hazardous Materials, 2015, 300: 398-405.

[ 2 ] Sun Y, Wang S, Niu J. Microbial community evolution of black and stinking rivers during in situ remediation through micro-nano bubble and submerged resin floating bed technology. Bioresource Technology, 2018, 258: 187-194.

[ 3 ] Ruiz-González C, Niño-García JP, Del Giorgio PA. Terrestrial origin of bacterial communities in complex boreal freshwater networks. Ecology Letters, 2015, 18(11): 1198-1206.

[ 4 ] Cheng W, Zhang J, Wang Z et al. Bacterial communities in sediments of a drinking water reservoir. Annals of Microbiolo$g y, 2014,64(2): 875-878$.

[ 5 ] Hale RL, Turnbull L, Earl SR et al. Stormwater infrastructure controls runoff and dissolved material export from arid urban watersheds. Ecosystems, 2015, 18(1) : 62-75.

[ 6 ] Tang Y, Li M, Zou Y et al. Mechanism of aerobic denitrifiers and calcium nitrate on urban river sediment remediation. International Biodeterioration \& Biodegradation, 2018, 126: 119-130.

[ 7 ] Gao H, Xie Y, Hashim S et al. Application of microbial technology used in bioremediation of urban polluted river: A case study of Chengnan river, China. Water, 2018, 10(5): 643.

[ 8 ] Jaynes ML, Carpenter SR. Effects of vascular and nonvascular macrophytes on sediment redox and solute dynamics. Ecology, 1986, 67(4): 875-882.

[ 9 ] Chen R, Barko JW. Effects of freshwater macrophytes on sediment chemistry. Journal of Freshwater Ecology, 1988, 4(3): 279-289.

[10] Nogales B, Lanfranconi MP, Piña-Villalonga JM et al. Anthropogenic perturbations in marine microbial communities. FEMS Microbiology Reviews, 2011, 35(2) : 275-298.

[11] Sun MY, Dafforn KA, Johnston EL et al. Core sediment bacteria drive community response to anthropogenic contamination over multiple environmental gradients. Environmental Microbiology, 2013, 15(9) : 2517-2531.

[12] Liu XW, Xie DP, Li KM et al. Influence of application of bio-energizer to sediment microbial community and the content of nitrogen and phosphorus element. China Environmental Science, 2013, 33(S1) : 87-92. [ 刘晓伟, 谢丹平, 李开明等. 投加生物促生剂对底泥微生物群落及氮磷的影响. 中国环境科学, 2013, 33( S1) : 87-92.]

[13] Liu T, Yuan J, Dong W et al. Effects on inorganic nitrogen compounds release of contaminated sediment treatment with in situ calcium nitrate injection. Environmental Science and Pollution Research, 2015, 22(2) : 1250-1260.

[14] Liu X, Tao Y, Zhou K et al. Effect of water quality improvement on the remediation of river sediment due to the addition of calcium nitrate. Science of the Total Environment, 2017, 575: 887-894.

[15] Gao J, Xiong Z, Zhang J et al. Phosphorus removal from water of eutrophic Lake Donghu by five submerged macrophytes. Desalination, 2009, 242(1/2/3) : 193-204.

[16] Lin JW, Zhu ZL, Zhao JF. Effect of calcium nitrate on cycling character of nutrient salt and organic matter between surface water and its sediments. Journal of Agro-environment Science, 2007, 26(1) : 58-63. [ 林建伟, 朱志良, 赵建夫. 硝酸钙 对底泥有机物及氮磷迁移循环的影响. 农业环境科学学报, 2007, 26(1): 58-63.]

[17] Lu LJ, Su YJ, Li XP et al. Application of bio-stimulant to the remediation of pollutant sediment. Environmental Science Survey, 2007, 26(6) : 49-53. [卢丽君, 孙远军, 李小平等. 用生物促生剂修复受污染底泥的试验研究. 环境科学 导刊, 2007, 26(6): 49-53.]

[18] Meagher RB. Phytoremediation of toxic elemental and organic pollutants. Current Opinion in Plant Biology, 2000, 3(2): 153-162.

[19] Huang XL, Guo YM, Wan B et al. Effect of submerged macrophytes restoration on the ecological environment of urban eutrophic lake. Environmental Engineering, 2018, 36(7): 17-21. [黄小龙, 郭艳敏, 万斌等. 沉水植物恢复对城市富营 养化湖泊生态环境影响. 环境工程, 2018, 36(7) : 17-21.] 
[20] He Z, Long X, Li L et al. Temperature response of sulfide/ferrous oxidation and microbial community in anoxic sediments treated with calcium nitrate addition. Journal of Environmental Management, 2017, 191: 209-218.

[21] Chen Y, Dai Y, Wang Y et al. Distribution of bacterial communities across plateau freshwater lake and upslope soils. Journal of Environmental Sciences, 2016, 43: 61-69.

[22] Xie Y, Wang J, Wu Y et al. Using in situ bacterial communities to monitor contaminants in river sediments. Environmental Pollution, 2016, 212: 348-357.

[23] Zheng G, Yampara-Iquise H, Jones J et al. Development of Faecalibacterium 16S rRNA gene marker for identification of human faeces. Journal of Applied Microbiology, 2009, 106(2) : 634-641.

[24] Knowles R. Denitrification. Microbiological Reviews, 1982, 46(1) : 43.

[25] Wang L, Zhang J, Li H et al. Shift in the microbial community composition of surface water and sediment along an urban river. Science of the Total Environment, 2018, 627: 600-612.

[26] Lee SH, Park JH, Kang HJ et al. Distribution and abundance of Spirochaetes in full-scale anaerobic digesters. Bioresource Technology, 2013, 145: 25-32.

[27] Jin S, Feng C, Tong S et al. Effect of sawdust dosage and hydraulic retention time (HRT) on nitrate removal in sawdust/ pyrite mixotrophic denitrification (SPMD) systems. Environmental Science: Water Research \& Technology, 2019, 5(2) : 346-357.

[28] Xu S, Yao J, Ainiwaer M et al. Analysis of bacterial community structure of activated sludge from wastewater treatment plants in winter. BioMed Research International, 2018: 8278970.

[29] Lovley DR. Dissimilatory Fe ( III ) and Mn ( IV ) reduction. Microbiology and Molecular Biology Reviews, 1991, 55(2) : 259-287.

[30] Kimbrough DE, Kouame Y, Moheban P et al. The effect of electrolysis and oxidation-reduction potential on microbial survival, growth, and disinfection. International Journal of Environment and Pollution, 2006, 27(1/2/3) : 211-221.

[31] Billen G. Etude de l'écométabolisme du mercure dans un milieu d'eau douce. Hydrobiological Bulletin, 1973, 7(2): 60-68.

[32] Stumm W. Redox potential as an environmental parameter; conceptual significance and operational limitation//3rd International Conference on Water Pollution Research, Section, 1966, 1: 1-16.

[33] Kralova M, Masscheleyn PH, Patrick Jr WH. Redox potential as an indicator of electron availability for microbial activity and nitrogen transformations in aerobic soil. Zentralblatt fiur Mikrobiologie, 1992, 147(6) : 388-399.

[34] Husson O. Redox potential ( Eh) and $\mathrm{pH}$ as drivers of soil/plant/microorganism systems: A transdisciplinary overview pointing to integrative opportunities for agronomy. Plant and Soil, 2013, 362(1/2) : 389-417.

[35] Zeng J, Yang LY, Liang Y et al. Spatial distribution of bacterial communities in sediment of a eutrophic lake revealed by denaturing gradient gel electrophoresis and multivariate analysis. Canadian Journal of Microbiology, 2008, 54 (12): 1053-1063. 